\title{
Kinetics and physiological implications of the growth behaviour of Eubacterium limosum on glucose/methanol mixtures
}

\author{
Pascal loubière, Evelyne Gros, $†$ Veronique Paquet $†$ and Nicholas D. Lindley* \\ Département de Génie Biochimique, CNRS UA 544, INSA, Complexe Scientifique de Rangueil, \\ F-31077 Toulouse Cédex, France
}

(Received 25 November 1991; revised 27 January 1992; accepted 12 February 1992)

\begin{abstract}
Batch-mode growth rates of the acetogenic anaerobe Eubacterium limosum on various substrates were dependent on the point of entry into the central metabolic network. During batch growth on glucose/methanol mixtures, simultaneous consumption of both substrates occurred at residual glucose concentrations below $6 \mathrm{mM}$ following an initial period of growth on glucose alone. During this mixotrophic growth phase, significantly higher biomass yields and specific growth rates were obtained than on either substrate alone. This phenomena was confirmed in chemostat experiments and stoichiometric analysis based on specific rates indicated that carbon and energy flow was significantly altered during mixotrophic growth with little net flux through the pyruvate ferredoxin oxidoreductase. It is suggested that such an effect on both growth rate and biomass yield was due to the improved availability of both carbon and energy for anabolic reactions.
\end{abstract}

\section{Introduction}

Mixed substrate conditions are widespread in natural ecosystems, in which the composition and relative concentrations of the substrate mixture have a profound influence on the metabolic activity of the constituent microbial population. To date, two modes of substrate utilization are regularly encountered dependent largely upon the substrate concentration. When substrate is in excess, sequential utilization of the carbon compounds tends to occur and a diauxic growth pattern is observed. The substrate that supports the highest growth rate is used preferentially and utilization of the second substrate is repressed (either at the level of the transport system or the enzymes specific to its metabolism) until the first substrate has been depleted. Alternatively, simultaneous use may take place under conditions in which the substrates are present at only low concentrations; conditions often encountered in natural habitats and relatively simple to model using a chemostat. Various authors have employed continuous cultures to study microbial behaviour on various substrate mixtures and growth rates (Brooke \& Attwood, 1983; Egli et al., 1980, 1983; Egli \& Lindley, 1984).

\footnotetext{
- Author for correspondence. Tel. 615599 88; fax 61559728.
}

† Present address: Sanofi Elf Bio-Recherches, 31328 Labege, France.
The following consequences of mixed substrate growth have been reported.

(1) An increase in growth yields from an assimilable substrate due to the use of a second substrate as energy source (Dijkhuizen \& Harder, 1979; Van Verseveld et al., 1979; Linton et al., 1981; Babel et al., 1983).

(2) Consumption of a second substrate at growth rates higher than the maximum rates obtained on this substrate alone providing that the first substrate supports more rapid growth than the second (Egli et al., 1982).

(3) Re-orientation of carbon flux within a metabolic network and in particular the shift in fermentation endproduct synthesis associated with anaerobic fermentation (Lindley et al., 1990).

In recent years mixed substrate utilization, from both biotechnological and physiological viewpoints has received increasing scientific interest though many aspects remain obscure. This report concerns the growth behaviour of an acetogenic anaerobe, Eubacterium limosum, whose fermentation characteristics are a function of the substrate presented. Growth on methylotrophic substrates is associated with high butyric acid yields and important intracellular concentrations of vitamin B12, whilst growth on glucose results in predominant production of acetate with $\mathrm{H}_{2}$ serving as the electron sink product. Specific growth rates are considerably faster on ghucose than on methanol. Such an organism provides a useful model for mixed substrate 
studies since not only are several distinct metabolic patterns associated with the choice of substrate, but a variety of substrates can be exploited which enter the central metabolic pathways at different sites. The results described illustrate that significant improvements can be made to growth dynamics if mixed substrate strategies are used to reinforce the carbon and energy flow downstream of major rate-limiting reactions.

\section{Methods}

Organism and growth conditions. The bacterium used was a strain of Eubacterium limosum initially isolated and identified by INRA (Villeneuve d'Ascq, France). The organism was cultivated under strict anaerobic conditions in $300 \mathrm{ml}$ and $800 \mathrm{ml}$ glass fermenters, or in sealed bottles. The medium used was that of Pacaud et al. (1985) containing $\mathrm{CO}_{2}\left(60 \mathrm{mM}\right.$, i.e. $5 \mathrm{~g} \mathrm{KHCO}_{3} \mathrm{H}^{-1}$ under $\left.15 \%, \mathrm{v} / \mathrm{v}, \mathrm{CO}_{2}\right)$ and acetate $(50 \mathrm{mM})$ to improve growth (Sharak-Genthner et al., 1981) as well as the growth-supporting carbon substrate(s). A suitably low redox potential was fixed by the aseptic addition of cysteine. $\mathrm{HCl}\left(0.5 \mathrm{~g} \mathrm{l}^{-1}\right)$ before inoculation. The $\mathrm{pH}$ was maintained at 7.4 using $\mathrm{NH}_{4} \mathrm{OH}$ and the incubation temperature was $37^{\circ} \mathrm{C}$. Inocula of $5 \%(\mathrm{v} / \mathrm{v})$ of a rapidly growing culture on the same media were used unless otherwise stated in the text.

Analytical methods. Growth rates were calculated from optical density measurements at $660 \mathrm{~nm}$ and biomass concentrations were estimated from a standard curve of optical density against dry weight.

Methanol and organic acids were determined by gas chromatographic methods after acidification of a culture filtrate with $\mathrm{HCl}$ and addition of an internal standard (propionic acid). A capillary column $(25 \mathrm{~m})$ containing Poraplot $\mathrm{Q}$ was used with flame ionization detection $\left(250^{\circ} \mathrm{C}\right)$. The oven temperature was increased progressively from 160 to $210^{\circ} \mathrm{C}$ at a rate of $10^{\circ} \mathrm{C} \mathrm{min}^{-1}$.

Gases were analysed chromatographically as described previously (Pacaud et al., 1985).

Glucose was analysed using an YSI-27A automatic analyser.

Fermentation balances. The substrate-product balances were assessed for the percentage recovery of major elements assuming that at $\mathrm{pH} 7.4$ organic acids were present exclusively as dissociated salts. A biomass formula of $\mathrm{C}_{4} \mathrm{H}_{7} \mathrm{O}_{2} \mathrm{~N}_{0.6}$, having a degree of reduction of 4.423 per carbon (Erickson, 1980) was used.

\section{Results}

\section{Growth on various substrates}

The maximum specific growth rates and fermentation end-product profiles were established for growth of $E$. limosum on a variety of simple carbon substrates entering the glycolysis/gluconeogenesis pathways at different sites (Table 1). The growth rates supported by each substrate were related to the proportion of carbon flow via glycolysis, glucose supporting the highest growth rate and methanol the lowest. The production of organic acids and $\mathrm{H}_{2}$ was also seen to be dependent on the substrate used (or the growth rate). During methylo-
Table 1. Growth rates and fermentation products of batch cultures of E. limosum on various substrates

\begin{tabular}{|c|c|c|c|c|c|}
\hline \multirow[b]{2}{*}{ Substrate } & \multirow{2}{*}{$\begin{array}{c}\text { Growth } \\
\text { rate } \\
\left(h^{-1}\right)\end{array}$} & \multicolumn{3}{|c|}{$\begin{array}{l}\text { Yields } \\
\text { (\% carbon) }\end{array}$} & \multirow{2}{*}{$\begin{array}{c}\mathbf{H}_{2} \\
\text { production }\end{array}$} \\
\hline & & Acetate & Butyrate & $\mathrm{CO}_{2}$ & \\
\hline Glucose & $0 \cdot 32$ & $30 \cdot 1$ & $6 \cdot 0$ & 32.9 & $++t$ \\
\hline Dihydroxyacetone & $0 \cdot 26$ & $40 \cdot 2$ & $24 \cdot 1$ & $9 \cdot 1$ & + \\
\hline Lactate & $0 \cdot 16$ & $16 \cdot 7$ & $62 \cdot 6$ & $5 \cdot 1$ & - \\
\hline Methanol/ $\mathrm{CO}_{2}$ & 0.08 & $35 \cdot 2$ & 81.4 & $-32 \cdot 2$ & - \\
\hline
\end{tabular}

trophic growth and also during growth on lactate, butyrate was the major end-product with no detectable $\mathrm{H}_{2}$ produced. For other substrates, acetate was the major product and reducing equivalent recycling took place predominantly by $\mathbf{H}_{2}$ production.

\section{Batch growth on glucose/methanol mixtures}

Glucose and methanol represent the opposing extremes as regards both growth and acetogenesis. Recent work relating to the methylotrophic growth of $E$. limosum on unicarbon substrates has shown that $\mathrm{CO}_{2}$ fixation associated with acetyl-CoA formation was rate-limiting, but could be overcome for brief periods during batch cultures by supplementing the medium with acetate (Loubière \& Lindley, 1991). However, the nature of the growth-rate-limiting reaction(s) has not yet been determined for glucose, though unconfirmed observations of transient pyruvate overflow under certain growth conditions (P. LeBloas, unpublished observations) may indicate that the reductive decarboxylation of pyruvate exerts a significant influence on the growth behaviour. If so, these limiting reactions are situated adjacently and the growth of the bacterium on glucose/methanol mixtures might be expected to be profoundly influenced.

When an equimolar carbon mixture was used $(50 \mathrm{mM}$ methanol $+8.3 \mathrm{~mm}$-glucose) the growth curve was made up of four phases (Fig. 1). During the first hours of the culture glucose was consumed alone supporting a specific growth rate of $0.31 \mathrm{~h}^{-1}$ as typically obtained for growth on glucose. The second phase of growth began when the residual glucose concentration had reached $6 \mathrm{~mm}$ (36 $\mathrm{mm}$-carbon), and represents the period in which simultaneous consumption of glucose and methanol took place associated with an increased growth rate $\left(\mu=0.4 \mathrm{~h}^{-1}\right)$. Specific rates of substrate consumption were highest during this phase $\left(4.8 \mathrm{mmol}\right.$ glucose $\mathrm{g}^{-1} \mathrm{~h}^{-1}$ and $12 \mathrm{mmol}$ methanol $\mathrm{g}^{-1} \mathrm{~h}^{-1}$ ) though interestingly, that of methanol diminished slightly (from 12 to $9 \mathrm{mmol} \mathrm{g}^{-1} \mathrm{~h}^{-1}$ ) throughout this phase of growth. Unfortunately, the gas phase analysis undertaken during 
this fermentation was not sufficiently precise to determine whether or not this decrease in methanol consumption was compensated by an increased fixation of $\mathrm{CO}_{2}$, such that the overall rate of synthesis of acetyl-CoA remained constant as might be expected from the constant growth rate. Following the complete depletion of glucose a halt occurred as regards growth even though a considerable concentration of residual methanol remained. After a lag phase lasting several hours growth restarted, as did consumption of methanol though specific rates were low. During this final phase of the culture, reconsumption of the $\mathrm{CO}_{2}$ and $\mathrm{H}_{2}$ produced during the period of glucose consumption also took place. Organic acid production was predominantly of acetate during the periods of heterotrophic and mixotrophic growth, but mostly of butyrate during the final phase of the culture. The averaged carbon conversion during the three phases in which growth occurred can be represented by the following stoichiometric equations (in $\mathrm{mM}$ ).

$$
\begin{gathered}
2.5 \text { Glucose } \rightarrow 1.5 \text { acetate }+0.5 \text { butyrate }+5 \text { cell } \mathrm{C} \\
+5 \mathrm{CO}_{2} \\
5.2 \text { Glucose }+8 \text { methanol }+1 \mathrm{CO}_{2} \rightarrow \\
7 \text { acetate }+1.5 \text { butyrate }+20 \text { cell } \mathrm{C} \\
15 \text { Methanol }+16 \mathrm{CO}_{2} \rightarrow 2 \text { acetate }+5.5 \text { butyrate } \\
+5 \text { cell } \mathrm{C}
\end{gathered}
$$

The overall fermentation can be represented by the following equation:

$$
\begin{aligned}
& 7.7 \text { Glucose }+23 \text { methanol }+12 \mathrm{CO}_{2} \vec{~} \\
& 10.5 \text { acetate }+7.5 \text { butyrate }+30 \text { cell } \mathrm{C}
\end{aligned}
$$

It can be seen that the period of double substrate growth resulted not only in a significantly improved growth rate but also a higher biomass yield relative to the totality of the substrate consumed than during growth on either glucose or methanol alone.

The phase of mixotrophic growth was initiated at a residual glucose concentration of approximately $6 \mathrm{mM}$, a value considerably higher than normally found. In order to determine whether or not the residual glucose really played a regulatory role fixing the onset of mixotrophy an experiment was undertaken in which the initial glucose concentration was set lower than this threshold value. In this fermentation the initial methanol concentration was as above but the glucose concentration was diminished to $4.2 \mathrm{mM}$. Simultaneous consumption of glucose and methanol was observed from the onset of growth. As previously, the growth rate during this phase was higher than that normally associated with growth on glucose alone. After the glucose had been totally consumed the fermentation was similar to that described above, with butyrate the predominant fermentation product once

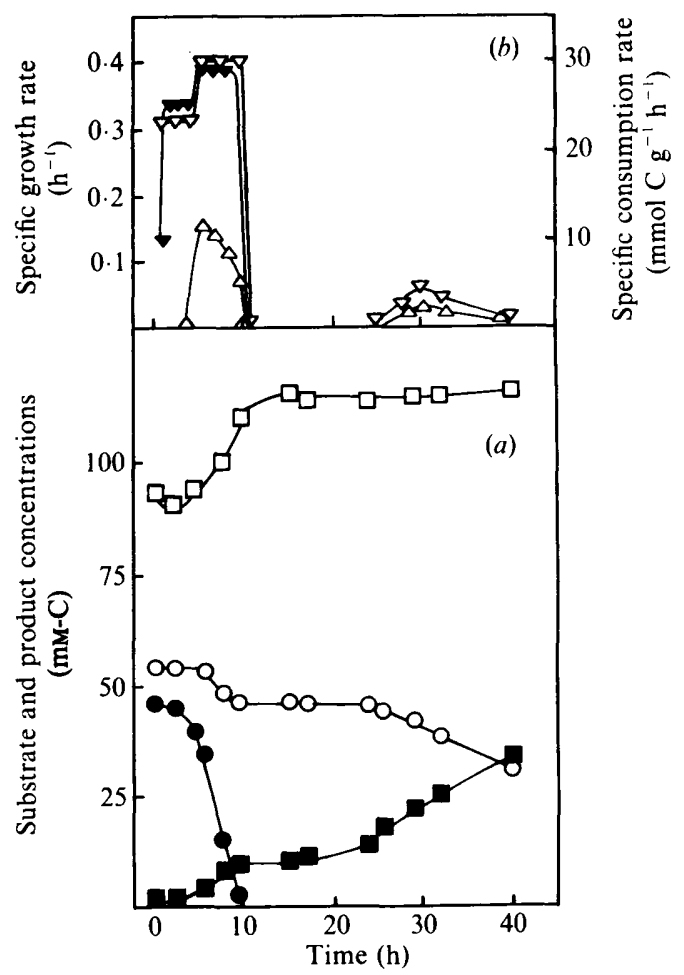

Fig. 1. Batch fermentation profile for the growth of E. limosum on a glucose/methanol (50/50, mM-carbon) mixture. (a) Substrate and product concentrations: $O$, glucose; $O$, methanol; $\square$, acetate; $\square$, butyrate. (b) Specific rates: of growth, $\nabla$; of glucose consumption, $\nabla$; and, of methanol consumption, $\Delta$.

metabolism of methanol restarted after a lag phase of 5-6 h. As with the previously described fermentation reconsumption of the $\mathrm{H}_{2}$ and $\mathrm{CO}_{2}$ produced during the initial growth period occurred in the later stages. By the end of the culture no trace of $\mathbf{H}_{2}$ could be detected in the gas phase.

Significantly higher initial glucose concentrations than the previous fermentations (i.e. $16.7 \mathrm{~mm}$ ) resulted in a classical diauxic growth pattern with no period of mixotrophy. Methanol consumption only began following a lag phase and even then at an extremely low rate. In this case, no detectable increase in biomass formation took place during the period of methanol consumption (and $\mathrm{H}_{2}+\mathrm{CO}_{2}$ reconsumption) but butyrate was produced.

\section{Chemostat growth on glucose}

The observations made during batch cultures were reexamined using continuous cultures in order to more precisely establish the mixotrophic behaviour of $E$. limosum. Although the growth of this organism has been described in continuous cultures with methanol as carbon source (Loubière \& Lindley, 1991) no information 


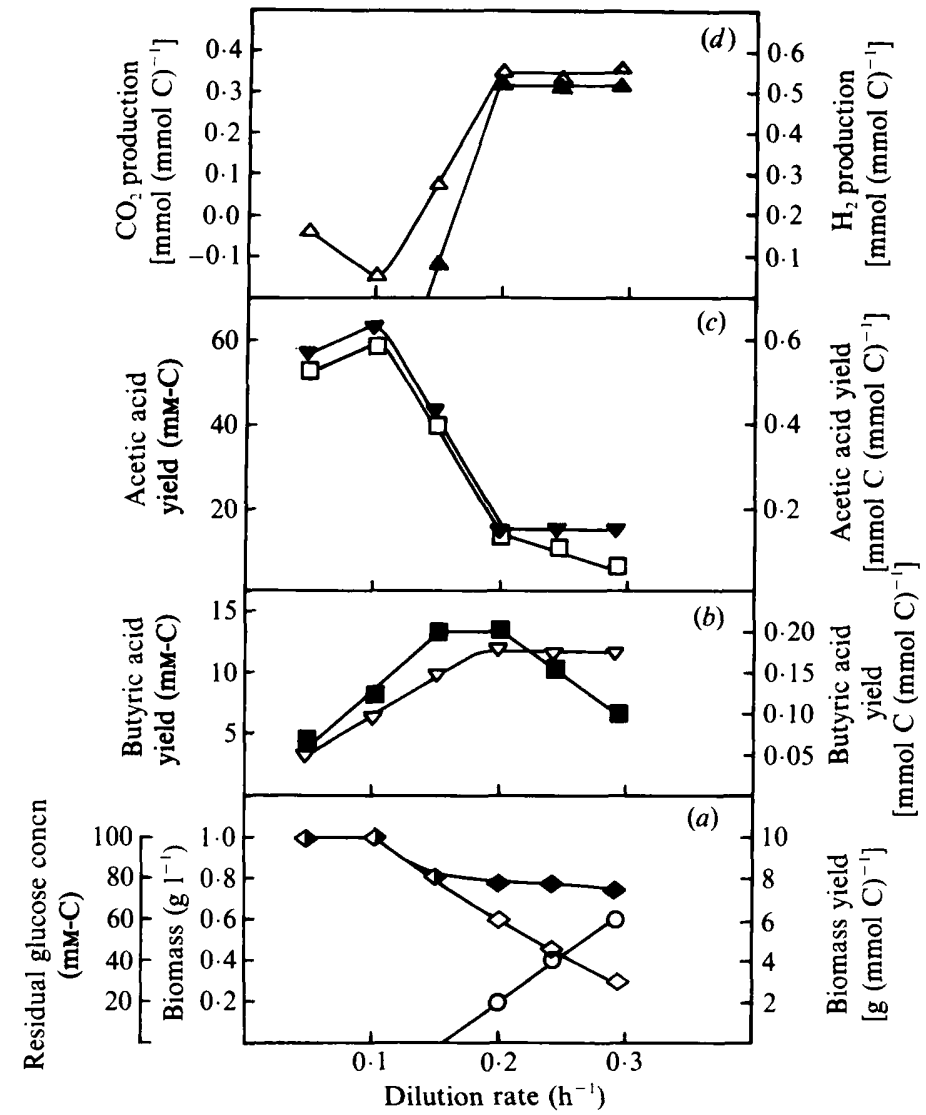

Fig. 2. Chemostat steady-state values for growth of $E$. limosum at various dilution rates on glucose. (a) $\bigcirc$, residual glucose concentration; $\diamond$, biomass concentration; $\bullet$, biomass yield. (b) $\square$, butyrate concentration; $\nabla$, butyrate yield. (c) $\square$, acetate concentration; $\nabla$, acetate yield. (d) $\triangle, \mathrm{CO}_{2}$ production or consumption (negative values); $\Delta, \mathbf{H}_{2}$ production. exists concerning the manner in which glucose is used. The growth, substrate consumption and production kinetics during growth on glucose at various growth rates are shown in Fig. 2. The highest steady-state exploited was at a dilution rate of $0.29 \mathrm{~h}^{-1}$ : wall growth and cell aggregation at higher rates made parameter estimation prone to error. Washout of all the non-attached biomass took place at a dilution rate of $0.4 \mathrm{~h}^{-1}$ indicating a maximum specific growth rate under chemostat conditions of between 0.29 and $0.4 \mathrm{~h}^{-1}$ as might be predicted from the biomass and residual substrate profiles. Residual glucose only appeared at growth rates in excess of $0 \cdot 16 \mathrm{~h}^{-1}$ and thereafter increased as a pseudo-linear function of the growth rate over the range of dilution rates interpreted. Supplementing the media with additional salts solutions did not alter the residual glucose profile. Biomass concentrations were related to the quantity of glucose consumed though some decrease was observed already at a dilution rate of $0.11 \mathrm{~h}^{-1}$. At dilution rates lower than this value no production of either $\mathrm{H}_{2}$ or $\mathrm{CO}_{2}$ was observed; indeed, some of the $\mathrm{CO}_{2}$ in the medium was consumed. At higher growth rates net production of both gases occurred, the $\mathrm{CO}_{2}$ corresponding to $33 \%$ of the substrate carbon consumed at the highest growth rates. The apparent shift in the organic acid product ratio can be explained by the variation in the $\mathrm{CO}_{2}$-fixing capacity. The more important production of acetate at low growth rates can be directly attributed to the capacity of $E$. limosum to produce acetate from the autotrophic pathway of $\mathrm{CO}_{2}$ assimilation occuring in acetogenic anaerobes. When at higher growth rates this autotrophic capacity ceases to function (for reasons as yet obscure) acetate production is diminished, though the mixed acid fermentation associated with the direct heterotrophic consumption of glucose remains largely the same.

\section{Chemostat growth on a glucose/methanol mixture}

An equimolar carbon mixture of glucose ( $8.3 \mathrm{~mm})$ and methanol $(50 \mathrm{mM})$ was used in order to investigate in more detail the behaviour of $E$. limosum during the mixotrophic growth mode. Acetate and $\mathrm{CO}_{2}$ supplements were as used previously. A variety of steady-states were established for a range of dilution rates up until $0.45 \mathrm{~h}^{-1}$, i.e. significantly higher than during growth on glucose alone. At higher growth rates, similar problems to those described for growth on glucose alone relating to 


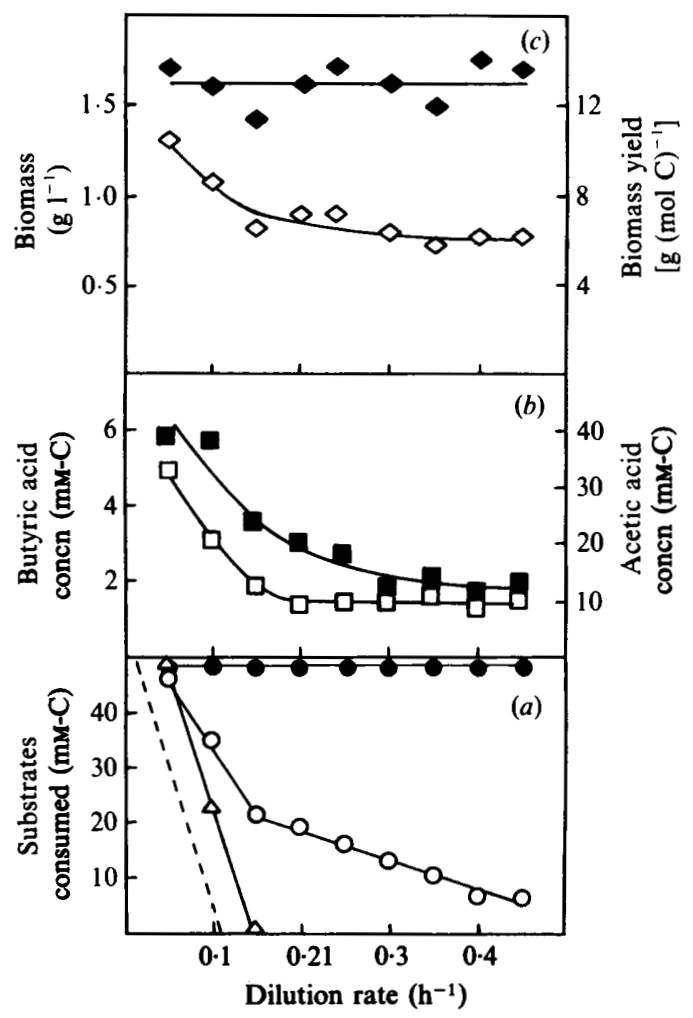

Fig. 3. Chemostat steady-state values for growth of $E$. limosum at various dilution rates on a glucose/methanol (50/50, mM-carbon) mixture. (a) $\bigcirc$, methanol consumption; $\triangle, \mathrm{CO}_{2}$ consumption; $\bullet$, glucose consumption. The dashed line represents methanol consumption under similar conditions but in the absence of glucose (from Loubière \& Lindley, 1991). (b) $\square$, acetate concentration; $\square$, butyrate concentration. (c) $\diamond$, biomass concentration; $\diamond$, biomass yield.

floc formation and wall growth were encountered making absolute determination of the maximum specific growth rate difficult $\left(\mu_{\mathrm{m}}\right.$ was situated between 0.45 and $0.60 \mathrm{~h}^{-1}$ ). The profile of substrate consumption was different to that obtained on single substrates (Fig. 3). No trace of residual glucose was observed under any of the steady-states. All the $\mathrm{H}_{2}$ and $\mathrm{CO}_{2}$ production normally associated with glucose catabolism was absent even at high growth rates and some consumption of the exogenous $\mathrm{CO}_{2}$ supplement occurred at low growth rates. The characteristics of methanol consumption was also stimulated, consumption being observed at all growth rates, with a displacement of the residual methanol curve towards more rapid growth rates compared to that obtained on methanol alone (see Loubière \& Lindley, 1991 : dashed line in Fig. 3). Above a dilution rate of $0.15 \mathrm{~h}^{-1}$ the increase in residual methanol deviated from the anticipated profile, the bacterium maintaining a constant specific consumption rate of $4.5 \mathrm{mmol}$ methanol $\mathrm{g}^{-1} \mathrm{~h}^{-1}$ and hence a linear increase in residual methanol. Presumably, the total consumption of glucose would cease when methanol consumption fell close to zero with an extremely abrupt washout hidden by the cell immobilization phenomenon. The ratio of organic acids produced did not show much variation.

\section{Discussion}

The maximum specific growth rate for a micro-organism on a carbon substrate is defined by the rate-limiting step(s) of its metabolism under the precise environmental conditions of growth. Comparison of the maximum specific growth rates obtained for $E$. limosum on various substrates shows that the point of entry into the central metabolic pathways to some extent determines the growth rate. In general, the greater the proportion of the flux using gluconeogenesis the less rapid the growth, most rapid growth being obtained for glucose whose catabolism is uniquely glycolytic. The maximum specific growth rate in batch fermentations on glucose alone was $0.31 \mathrm{~h}^{-1}$, but presumably many of the enzymes were capable of supporting a more rapid rate. If the carbon flux can be restored after rate-limiting reactions, improved growth rates should be obtained. In the case of simultaneous utilization of glucose/methanol mixtures, methanol enters central metabolism as acetyl-CoA and reinforces the carbon flux immediately downstream of the pyruvate:ferredoxin oxidoreductase. In view of the relative rates of each substrate's consumption net gluconeogenesis from the unicarbon substrate can be discounted, suggesting that the reductive decarboxylation of pyruvate may be a rate-limiting reaction for growth on glucose alone.

The residual glucose profile obtained in chemostat cultures grown on glucose (Fig. 2) might be taken to indicate that the cultures were limited by factors other than the carbon source at high growth rates. Since supplementing the medium with individual salts, or complete salts mixtures, did not alter the profile, nutritional limitations can be ruled out. Similar kinetic behaviour can also be obtained for cultures in which endproduct inhibition increases the energy demand. In the case of $E$. limosum the situation is more complex since certain acetogenic bacteria are able to grow autotrophically obtaining a net gain in energy during the transformation of $\mathrm{H}_{2} / \mathrm{CO}_{2}$ or $\mathrm{CO}$ to acetate via the intermediary of the proton-motive force (Heise et al., 1989). However, since this strain cannot grow on such substrates, or on formate, but is able to transform them to acetate (Loubière et al., 1987), such a gain in energy can probably be ignored. Thus, the growth kinetics can most likely be attributed to increased energy expenditure related to the accumulation of butyrate or of $\mathbf{H}_{2}$. Further work to analyse growth efficiency would enable this point to be clarified. 
The scientific literature contains relatively few examples of this growth-rate-enhancing effect during growth on mixed substrates, though in all cases the effect was due to reinforced flux to overcome metabolic bottlenecks. This was certainly the case for glucose/galactose mixtures for growth of Thiobacillus A2 in which sugar transport during growth on glucose alone was a severe limitation on the ensuing glycolytic flux (Wood \& Kelly, 1977). In a somewhat different approach, Robertson \& Kuenen (1984) obtained a growth-rate-stimulating effect by using mixtures of respiratory electron acceptors.

In the present study both growth rates and biomass yields have been improved simultaneously. This implies that the modified carbon flow pattern resulting from mixotrophic metabolism must have overcome not only the rate at which anabolic precursors were supplied, but also the energy production necessary to assimilate an increased proportion of these metabolites. The net energy yield associated with the catabolic pathways of $E$. limosum can be easily estimated from stoichiometric data (assuming that ATP production is associated uniquely with substrate level phosphorylation reactions). An analysis of this type shows that anabolic efficiency remains constant and the increased biomass yield was directly related to the improved production of ATP. Such a finding is logical and in accord with previous reports concerning the potential gain in biomass yields from the utilization of an auxiliary substrate as an additional energy source (Babel et al., 1983). For E. limosum the improved biomass yield was unambiguous, being related to the totality substrate consumed and not merely the glucose [see review of Linton (1990) concerning this subject]. Whilst such a finding would appear to be logical it is somewhat surprising that such a close agreement between biomass yield and ATP production was found since this implies that the biomass yield was limited by the available energy, apparently in contradiction with the calculations of Papoutsakis (1984). This author developed a modelling approach for predicting carbon and energy availability during growth of the butyric acid bacteria on glucose. He concluded that during the acidogenic phase ATP was in large excess. However, such an approach failed to take into account the ATPconsuming reactions not directly associated with the growth (i.e. maintenance requirements) which have recently been shown to be a significant drain on the energy reserves for this type of fermentation (Loubière \& Lindley, 1991).

During batch growth on glucose/methanol mixtures the pattern of substrate consumption was somewhat unusual. The phase of mixotrophic growth induced at residual glucose concentrations of $6 \mathrm{mM}$ cannot be explained by enzyme induction or derepression effects, this threshold value being considerably higher than that normally associated with such effects (e.g. Brooke \& Attwood, 1983). Furthermore, exactly the same profiles were obtained for inocula pre-grown on either methanol or glucose as sole substrates. It would appear therefore that methanol consumption is delayed because of a biochemical inhibition of one or more enzymes involved in the acetyl-CoA condensation pathway. The nature of this control remains obscure and will require a detailed study of the enzyme profiles involved. The lag phase occurring between the end of the mixotrophic growth phase due to glucose depletion and the onset of methylotrophic metabolism can be explained by the need to control the gluconeogenic flux: certain enzymes will need to be induced (e.g. fructose bisphosphatase) and others diminished if futile cycles are to be avoided.

At first sight the results obtained for batch growth on glucose/methanol $(100: 50)$ are in contradiction with the other results since no period of mixotrophic growth took place. However, this apparent anomaly can be explained by the inhibitory effects of the growth environment on the methylotrophic growth. Under conditions of high organic acid concentrations growth on methanol would be severely inhibited, as shown by the absence of significant biomass formation during the methylotrophic phase of the fermentation. During this phase, rates of methanol consumption were low. Bearing in mind the relatively short period of the fermentation susceptible to promote mixotrophic growth and the diminished capacity to consume methanol it would seem unlikely that any significant data could be obtained. Thus, it is possible that a brief period of mixotrophic metabolism may have occurred though of greatly reduced magnitude. This may explain why other groups have not observed this effect (Sharak-Genthner \& Bryant, 1987).

The use of methanol together with glucose results in an additive effect of both growth rates and biomass yields, implying that the perturbation such a nutritional regime imposes overcomes not only carbon flow bottlenecks but also provokes an improved production of biochemical energy. Whilst it would seem likely that during mixed substrate growth there occurs a partition of carbon flux, such that the methanol is predominantly incorporated into the organic acid fermentation products, isotopic tracer studies are necessary to confirm this. However, supportive evidence of this hypothesis can be seen in the manner in which gas phase composition during mixotrophic growth is notable by the quasi-absence of $\mathrm{H}_{2} \mathrm{O}$. This could be interpreted in conjunction with the stoichiometric data as being indicative of a greatly reduced flux through the pyruvate :ferredoxin oxidoreductase. Further research should concentrate on the role of this enzyme in determining the fermentation behaviour of acetogenic anaerobes. 


\section{References}

BABEL, W., MÜlLER, R. H. \& MARKuSKe, K. D: (1983). Improvement of growth yield of yeast on glucose to the maximum by using an additional energy source. Archives of Microbiology 136, 203-208.

BROOKE, A. G. \& ATTWOOD, M. M. (1983). Regulation of enzyme synthesis during the growth of Hyphomicrobium $\mathrm{X}$ on mixtures of methylamine and ethanol. Journal of General Microbiology 129, 2399 2404.

DIJKHUIZEN, L. \& HARDER, W. (1979). Growth on mixtures of acetate and formate in continuous culture. Archives of Microbiology 123, $47-53$.

EgLI, T. \& Lindley, N. D. (1984). Mitochondrial activities in the methylotrophic yeast Kloeckera sp.2201 during growth with glucose and/or methanol. Journal of General Microbiology 130, 3239-3249.

Egli, T., van Dijken, J. P., Veenhuis, M., Harder, W. \& Fiechter, A. (1980). Methanol metabolism in yeasts: regulation of the synthesis of catabolism enzymes. Archives of Microbiology 124, 115-121.

EGLI, T., KÄPPELI, O. \& FIECHTER, A. (1982). Regulatory flexibility of methylotrophic yeasts in chemostat cultures: simultaneous assimilation of glucose and methanol at a fixed dilution rate. Archives of Microbiology 131, 1-7.

Egli, T., Lindley, N. D. \& Quayle, J. R. (1983). Regulation of enzyme synthesis and variation of residual methanol concentration during carbon-limited growth of Kloeckera sp.2201 on mixtures of methanol and glucose. Journal of General Microbiology 129, 12691281.

EricKson, L. E. (1980). Biomass elemental composition and energy content. Biotechnology and Bioengineering 22, 451-453.

Heise, R., Muller, V. \& GotTschalK, G. (1989). Sodium dependence of acetate formation by the acetogenic bacterium Acetobacterium woodii. Journal of Bacteriology 171, 5473-5478.

Lindley, N. D., Durand, G. \& Soucaille, P. (1990). Contrôle du flux du carbone chez les anaérobies utilisant des mélanges de substrats. In Stratégie d'Utilisation des Substrats pour la Production de Métabolites Microbiens, pp. 113-128. Edited by L. M. Lebeault \& J. P. Pan. Paris: SFM.
Linton, J. D. (1990). Potential of dual substrates for biomass and metabolite production. In Mixed and Multiple Substrates and Feedstocks, pp. 33-44. Edited by G. Hamer, T. Egli \& M. Snozzi. Konstanz, Germany: Hartung-Gorre-Verlag.

Linton, J. D., Griffiths, K. \& GREGORY, M. (1981). The effect of mixtures of glucose and formate on the yield and respiration of a chemostat culture of Beneckea natriegens. Archives of Microbiology 129, 119-122.

Loubière, P. \& Lindley, N. D. (1991). The use of acetate as an additional co-substrate improves methylotrophic growth of acetogens when $\mathrm{CO}_{2}$ fixation is rate-limiting. Journal of General Microbiology 137, 2247-2251.

Loubière, P., Pacaud, S., Goma, G. \& Lindley, N. D. (1987). The effect of formate on the acidogenic fermentation of methanol by Eubacterium limosum. Journal of General and Applied Microbiology 33, 463-470.

PaCaud, S., Loubière, P. \& Goma, G. (1985). Methanol metabolism by Eubacterium limosum $\mathrm{B} 2$ : effects of $\mathrm{pH}$ and carbon dioxide on growth and organic acid production. Current Microbiology 12, 245250.

Papoutsakis, E. T. (1984). Equations and calculations for fermentations of butyric acid bacteria. Biotechnology and Bioengineering 26, 174-187.

Robertson, L. A. \& Kuenen, J. G. (1984). Aerobic denitrification: a controversy revived. Archives of Microbiology 139, 351-354.

Sharak-Genthner, B. R., Davis, C. L. \& Bryant, M. P. (1981). Features of rumen and sewage sludge strains of Eubacterium limosum, a methanol and $\mathrm{H}_{2}-\mathrm{CO}_{2}$-utilizing species. Applied and Environmental Microbiology 42, 12-19.

Van Verseveld, H. D., Boon, J. P. \& Stouthamer, A. H. (1979). Growth yields and the efficiency of oxidative phosphorylation of Paracoccus denitrificans during two-(carbon)substrate limited growth. Archives of Microbiology 121, 213-223.

WoOD, A. P. \& Kelly, D. P. (1977). Heterotrophic growth of Thiobacillus A2 on sugars and organic acids. Archives of Microbiology $113,257-264$. 